American Journal of Animal and Veterinary Sciences 7 (2): 67-74, 2012

ISSN 1557-4555

(C) 2012 Science Publications

\title{
Plasma Fatty Acid Profile of Gestating Ewes Supplemented with Fishmeal
}

\author{
Mamun M. Or-Rashid, Rebecca Fisher, \\ Niel Karrow, Ousama Al Zahal and Brian W. McBride \\ Department of Animal and Poultry Science, \\ University of Guelph, Guelph, Ontario N1G 2W1, Canada
}

\begin{abstract}
Problem statement: The very long chain n-3 polyunsaturated fatty acids (>18C) cannot be adequately synthesized by ruminant tissues to meet their requirements; therefore, their concentration in body depends on the supply through feed. It may be possible to improve the essential fatty acid status of ruminant animals, during gestation by manipulating the maternal diet with fishmeal (FM). The objectives of this research were to (1) determine the effect of fishmeal supplementation on the plasma fatty acid profile of ewes during late gestation and (2) determine the status of the plasma docosahexaenoic acid (22:6n3) of lambs born to these ewes. Approach: Eight gestating ewes [RideauArcott, $97 \pm 5 \mathrm{~kg}$ initial body weight, 100 days of gestation] were used in a completely randomized design. Ewes were individually-housed and fed either a control diet (supplemented with soybean meal) or a fishmeal supplemented diet. Blood samples were collected via jugular venipuncture for plasma fatty acids analysis on 100, 114, 128 and 142 days of gestation after morning feeding. Blood samples from the lambs were also collected via jugular venipuncture immediately after birth and before receiving their mothers' colostrum. Plasma fatty acids were analyzed by gas-liquid chromatography. Results: The ewes from both groups, i.e., control and fishmeal supplemented, had a similar fatty acid profile prior to supplementation (at 100 days, $\mathrm{p}>0.05$ ). Thereafter, there was an increase in eicosapentaenoic acid (20:5n3), docosahexaenoic acid, total n3-PUFA and total very long chain n3PUFA ( $>$ C18) contents in plasma for the fishmeal supplemented ewes compared to the control $(\mathrm{p}<0.03)$. There were no differences $(\mathrm{p}>0.05)$ in total saturated fatty acids, total monounsaturated fatty acids, total conjugated linoleic acid, total trans-18:1, total cis-18:1, or total n6-PUFA contents in ewe plasma between control and fishmeal supplemented groups. Lambs born to ewes fed the fishmeal supplemented diet had greater $(\mathrm{p}<0.05)$ plasma concentrations of eicosapentaenoic acid $(0.7 \mathrm{Vs} 0.4$, g/100 g FA), docosahexaenoic acid (1.6 Vs 0.9, g/100 g FA) and total very long chain n3-PUFA (3.3 vs. 2.0, g/100 g FA) than lambs born to ewes fed the control diet. Conclusion: The ewes supplemented with fishmeal supplementation showed a positive response with the enrichment of docosahexaenoic acid, eicosapentaenoic acid and total very long chain n3-PUFA in plasma during gestation and these fatty acids were transferred to the fetus as well.
\end{abstract}

Kew words: Docosahexaenoic acid, Eicosapentaenoic acid, Fishmeal, Very long chain n3-PUFA, Ewe plasma, Gestation

\section{INTRODUCTION}

The very long chain n-3 polyunsaturated fatty acids (>18C, VL_n3-PUFA), such as, docosahexaenoic acid (DHA, 22:6n3), are essential for normal growth and development in mammals (Williams, 2000). A specific functional role of DHA had been shown in the fetus and early infant neural development, which might influence neonatal viability (Cattaneo et al., 2006; Capper et al., 2007). Docosahexaenoic acid is highly concentrated in brain tissues and selectively accumulated during later stages of fetal development and infant brain growth (Brenna and Lapillonne, 2009; Hsieh and Brenna, 2009). Research suggests that DHA intake in pregnancy provides advantages to both the developing fetus and mother (Saldanha et al., 2009). A major factor contributing to high lamb mortality rates in the sheep industry is hypothermia, due to delayed suckling and exhaustion of brown fat reserves (Singer, 1998). Studies in sheep have shown that supplementation of diets with fish oil, a source of VL_n3-PUFA, during late pregnancy reduced the latency of suckling in lambs (Capper et al., 2006).

Corresponding Author: Mamun M. Or-Rashid, Department of Animal and Poultry Science, University of Guelph, Guelph, Ontario N1G 2W1, Canada 
It is well documented that the essential fatty acid (FA) status of ruminant animals at birth is less than satisfactory (Noble et al., 1978). On the other hand, the VL_n3-PUFA cannot be adequately synthesized by ruminant tissues to meet their requirements; therefore, their concentration in body depends on the supply through feed. It may be possible to improve the essential fatty acid status of ruminant animals, during gestation by manipulating the maternal diet with Fishmeal (FM). The purposes of this study were to 1) determine the effect of FM-supplementation on the plasma FA profile of ewes during late gestation and 2) determine the status of plasma FA of lambs born to these ewes.

\section{MATERIALS AND METHODS}

All experimental procedures were done with the approval of the University of Guelph Animal Care Committee in accordance with the guidelines of the Canadian Council on Animal Care (CCAC, 1993).

Animals and experimental treatments: Eight gestating ewes [Rideau-Arcott, $97 \pm 5 \mathrm{~kg}$ initial body weight, 100 days (d) of gestation] of similar nutritional and environmental background, were obtained from Ponsonby Sheep Research Station (University of Guelph, Ontario, Canada) and individually-housed in 4 by 6 foot indoor pens and randomly assigned to either a control diet (supplemented with soybean meal) or a FMsupplemented diet $(2.64 \mathrm{~kg} / \mathrm{day}$ as fed, comprising $0.312 \mathrm{~kg}$ protein-supplement, $0.441 \mathrm{~kg}$ mixed grain, $0.630 \mathrm{~kg}$ chopped hay, $1.261 \mathrm{~kg}$ alfalfa pellets). Ingredient composition of the diets is presented in Table 1. The nutrient requirements were based on both the body weight and gestational stage of the ewes determined by the Cornell Net Carbohydrate and Protein System for Sheep (Cornell University, Ithaca, NY). Feed was offered twice a day at 8:00 a.m. and 4:00 p.m., with orts being collected before the morning feeding. Animals were given ad libitum access to water throughout the duration of the study. The pooled samples of the experimental diets were analyzed for dry matter content by drying in an oven at $60^{\circ} \mathrm{C}$ for $48 \mathrm{~h}$ (AOAC, 1996). A subsample was ground using a Wiley Mill with a 1-mm screen (Thomas-Wiley, Philadelphia, PA) and stored at $-20^{\circ} \mathrm{C}$ until analyzed. Samples were analyzed at a commercial laboratory (Agri-Food Laboratory,
Guelph, Ontario, Canada) for crude protein, crude fat, lignin, acid detergent fiber and neutral detergent fiber.

Blood sampling: Blood samples from the ewes were collected via jugular venipuncture on 100, 114, 128 and 142 day of gestation after morning feeding using heparin vacutainers (Becton, Dickinson and Company, Franklin Lks, NJ). Blood samples from the lambs were also collected via jugular venipuncture immediately after birth and before receiving their mothers' colostrum. Blood samples were centrifuged at $500 \times \mathrm{g}$ for $15 \mathrm{~min}$ to separate the plasma. The plasma samples were kept at $-20^{\circ} \mathrm{C}$ for subsequent fatty acids analysis.

Analysis of samples for fatty acids: Total lipid in plasma was extracted according to the method by Bligh and Dyer (1959) with minor modifications. A $0.20 \mathrm{~mL}$ of plasma was taken in a $15-\mathrm{mL}$ screw-top culture tube with Teflon cap and mixed with water to a total vol of $1 \mathrm{~mL}$; then $2.5 \mathrm{~mL}$ of methanol and $1.25 \mathrm{~mL}$ of chloroform were added in the same tube and vortexed. The contents of the culture tube were kept at room temperature for $60 \mathrm{~min}$ with every 10 min vortexing. After $1 \mathrm{~h}, 1.25 \mathrm{~mL}$ of chloroform, $1.15 \mathrm{~mL}$ of water and $0.1 \mathrm{~mL}$ of $3 \mathrm{M} \mathrm{HCl}$ were added, vortexed and centrifuged at $1,200 \times g$ for $3 \mathrm{~min}$ at room temperature. The acid (i.e., $3 \mathrm{M} \mathrm{HCl}$ ) was added to ensure the $\mathrm{pH}$ of the extract was acidic. The chloroform layer (bottom phase) containing lipid was removed using 2 Pasteur pipettes, one inserted into other. The methanol-water phase was extracted with an additional $1.25 \mathrm{~mL}$ of chloroform and the chloroform phases were combined, dried over anhydrous $\mathrm{Na}_{2} \mathrm{SO}_{4}$, filtered and then transferred into a 4-mL vial. Chloroform was removed from the vial under a stream of $\mathrm{N}_{2}$ and 3 drops of benzene were added and vortexed. The lipid content in the vial was methylated by adding $200 \mu \mathrm{L}$ of $\mathrm{NaOCH}_{3}(0.5 \mathrm{M}$ solution in methanol, Sigma-Aldrich, St. Louis, MO) (Cruz-Hernandez et al., 2004; Muller et al., 2005). The vials were kept at room temperature for $25 \mathrm{~min}$. Then, $1 \mathrm{~mL}$ of $1 \mathrm{~N}$ methanolic sulfuric acid $(2.8 \mathrm{~mL}$ of $96 \%$ sulfuric acid in $100 \mathrm{~mL}$ methanol) was added. After vortexing, the vials were heated at $50^{\circ} \mathrm{C}$ for $15 \mathrm{~min}$. After cooling at $-20^{\circ} \mathrm{C}$ for $3 \mathrm{~min}, 1.0 \mathrm{~mL}$ of water and $1.0 \mathrm{~mL}$ of hexane were added, vortexed and centrifuged. The upper portion (i.e., hexane layer) containing Fatty Acid Methyl Esters (FAME) was transferred into another vial for GLC analysis. 
American J. Animal \& Vet. Sci., 7 (2): 67-74, 2012

Table 1: Ingredients and chemical analysis of experimental diets

\begin{tabular}{|c|c|c|}
\hline \multirow[b]{2}{*}{ Ingredients } & \multicolumn{2}{|c|}{ Dry matter $(\%)$} \\
\hline & Control & $\begin{array}{l}\text { Fishmeal } \\
\text { supplemented }\end{array}$ \\
\hline $\begin{array}{l}\text { Mixed grain (barley, } \\
\text { oats and corn) }\end{array}$ & 16.34 & 16.34 \\
\hline Alfalfa hay & 24.72 & 24.72 \\
\hline Alfalfa pellets & 47.26 & 47.26 \\
\hline \multicolumn{3}{|l|}{ Protein supplement } \\
\hline Soybean meal & 6.62 & -- \\
\hline Fishmeal & -- & 4.73 \\
\hline Feather meal & 2.36 & 1.47 \\
\hline Wheat grain & 0.71 & 2.36 \\
\hline Wheat shorts & -- & 1.68 \\
\hline $\begin{array}{l}\text { Calcium mono } \\
\text { phosphate }\end{array}$ & 0.66 & 0.25 \\
\hline Calcium carbonate & 0.57 & 0.42 \\
\hline Salt & 0.42 & 0.38 \\
\hline Magnesium oxide & 0.19 & 0.23 \\
\hline Minerals and vitamines ${ }^{1}$ & 0.16 & 0.16 \\
\hline \multicolumn{3}{|l|}{ Chemical composition } \\
\hline Dry matter $(\%)$ & 90.70 & 90.70 \\
\hline Crude protein $(\mathrm{N} \times 6.25)$ & 21.60 & 21.60 \\
\hline A Neutral detergent fiber & 22.70 & 22.50 \\
\hline Neutral detergent fiber & 35.30 & 36.30 \\
\hline Lignin & 4.70 & 4.80 \\
\hline Crude fat & 2.60 & 2.80 \\
\hline $\mathrm{NFC}^{2}$ & 37.60 & 37.10 \\
\hline $\mathrm{ME}^{3}, \mathrm{Mcal} / \mathrm{kg}$ & 2.10 & 2.10 \\
\hline
\end{tabular}

${ }^{1}$ Floradale Feed Mill Limited, Floradale, ON, Canada; ${ }^{2}$ Nonfiber carbohydrates $=100-[$ (neutral detergent fiber - neutral detergent insoluble protein $)+$ crude protein + crude fat + ash] ${ }^{3}$ Calculated using the Cornell Net Carbohydrate and Protein System for sheep (CNCPSSheep v. 1.0.21, Cornell University, Ithaca, NY) using forage chemical analysis.

Table 2: The fatty acid composition of experimental diets (Dry matter basis

\begin{tabular}{lll}
\multicolumn{1}{c}{ matter basis $)$} & & \\
\hline & (g / kg total Dry matter $)$ \\
& - Fishmeal & Fatty acids \\
Control supplemented & & \\
$10: 0$ & 0.04 & 0.04 \\
$12: 0$ & 0.09 & 0.10 \\
$14: 0$ & 0.23 & 0.32 \\
$14: 1$ & 0.01 & 0.01 \\
$16: 0$ & 5.39 & 5.75 \\
$9 c-16: 1$ & 0.17 & 0.25 \\
$18: 0$ & 0.93 & 0.89 \\
$9 c-18: 1$ & 2.44 & 2.39 \\
$11 c-18: 1$ & 0.24 & 0.30 \\
$18: 2 \mathrm{n} 6$ & 7.02 & 6.98 \\
$20: 0$ & 0.29 & 0.30 \\
$20: 1$ & 0.05 & 0.09 \\
$18: 3 \mathrm{n} 3$ & 8.51 & 9.35 \\
$22: 0$ & 0.28 & 0.30 \\
$20: 4 \mathrm{n} 6$ & 0.01 & 0.02 \\
$20: 5 \mathrm{n} 3$ & nd & 0.20 \\
$24: 0$ & 0.31 & 0.33 \\
$22: 5 \mathrm{n} 3$ & nd & 0.02 \\
$22: 6 \mathrm{n} 3$ & nd & 0.36 \\
\hline
\end{tabular}

nd: not detected
The hexane containing FAME was analyzed by GLC and subsequently identified as described by CruzHernandez et al. (2004) and Odongo et al. (2007) with a different temperature program. Briefly, FAME analysis was performed using an Agilent 6890N GLC (Agilent Technologies, Palo Alto, CA) equipped with a split-splitless injector at $250^{\circ} \mathrm{C}$, a flame-ionization detector at $250^{\circ} \mathrm{C}$ and a CP Sil 88 column $(100 \mathrm{~m} \times 0.25$ $\mathrm{mm}, \quad 0.2 \mu \mathrm{m}$ of film thickness; Varian Inc., Mississauga, ON, Canada). Hydrogen was used as the carrier gas at a constant flow rate of $1 \mathrm{~mL} / \mathrm{min}$. The temperature of the GLC oven was set to $45^{\circ} \mathrm{C}$ for $4 \mathrm{~min}$, increased at $13^{\circ} \mathrm{C} / \mathrm{min}$ to $173^{\circ} \mathrm{C}$ and held for $28 \mathrm{~min}$ and increased at the rate of $4{ }^{\circ} \mathrm{C} / \mathrm{min}$ to a final temperature of $215^{\circ} \mathrm{C}$ and held for $43 \mathrm{~min}$. Agilent Technologies Chemstation software (Rev. B.01.01) was used for data analysis. A 1- $\mu \mathrm{L}$ sample was injected at splitless mode. Peaks were routinely identified by comparison of retention times with fatty acid methyl ester standards (GLC \#463, \#UC-59-M, C21:0, C23:0 and C26:0; NuCheck Prep Inc., Elysian, MN). In addition, some peaks of 18:1 and CLA and branched-chain FA, for which standard FAME were not available, were identified by comparison to published data as described by Kramer et al. (2002), Cruz-Hernandez et al. (2004) and Odongo et al. (2007).

The samples from the experimental diets were also analyzed for FA as shown in Table 2. The FMsupplemented diet provided 20:5n3, 22:5n3 and 22:6n3 of $0.20 \mathrm{~g} / \mathrm{kg}$ total DM, $0.02 \mathrm{~g} / \mathrm{kg}$ total DM and 0.36 $\mathrm{g} / \mathrm{kg}$ total DM, respectively.

Statistical analysis: The data were analyzed as a completely randomized design using the PROC MIXED procedure of SAS (v. 9.1; SAS Inst., Inc., Cary, NC) using the model $Y_{\mathrm{ijk}}=\mu+\alpha_{\mathrm{i}}+\beta_{\mathrm{j}}+(\alpha \times \beta)_{\mathrm{ij}}+$ $\varepsilon_{\mathrm{ijk}}$, where $\mathrm{Y}_{\mathrm{ijk}}=$ the dependent variable, $\mu=$ overall mean, $\alpha_{\mathrm{i}}=$ effect of diet $(\mathrm{i}=1,2), \beta_{\mathrm{j}}=$ effect of day $(\mathrm{j}=$ $1,2,3,4),(\alpha \times \beta)_{\mathrm{ij}}=$ effect of diet by day interaction $\left(\mathrm{ij}_{\mathrm{ij}}=\right.$ $1,2,3,4,5,6,7,8)$ and $\varepsilon_{\mathrm{ijk}}=$ random residual error. The effects of diet and day were considered as fixed effects. Day of experiment was used as a repeated measurement with ewe within dietary treatment as the subject. For each analyzed variable, animal was subjected to five covariance structures: compound symmetry, heterogeneous compound symmetry, autoregressive order 1 , heterogeneous autoregressive order 1 and unconstructured. The covariance structure that gave the smallest Bayesian information criterion was used (Littell et al., 1996). Orthogonal polynomial contrast was used to describe the linear and quadratic terms of the day effect and diet by day interaction.

The FA contents of lamb plasma were analyzed as a completely randomized design using the PROC MIXED procedure of using the model $Y_{j}=\mu+\beta_{j}+\varepsilon_{j k}$; 
where $\mu=$ overall mean, $\beta=$ effect of diet $(j=1,2)$ and $\varepsilon_{\mathrm{jk}}=$ random residual error. Effects were considered significant at a probability of $\mathrm{p}<0.05$.

\section{RESULTS}

The mean percentages of the different FA in ewe plasma are given in Table 3-5. In the present study, the major FA in plasma of sheep were palmitic $(16: 0)$, stearic $(18: 0)$, oleic $(9 \mathrm{c}-18: 1)$, linoleic $(18: 2 n 6)$ and linolenic (18:3n3) acids. The ewes from both groups had a similar FA profile prior to supplementation (100 d, p>0.05, Table 3-5).

Among Saturated Fatty Acids (SFA), the percentages of 12:0, 13:0, 14:0, 15:0, 17:0, 21:0, 22:0, 23:0, 24:0 and 26:0 were not significantly different $(p>0.05)$ between the two experimental groups (Table 3). Among these FA, there was no diet by day $(100,114,128$ and $142 \mathrm{~d}$ of gestation) interaction effect ( $p>0.05)$; but some of them, such as 12:0, 14:0, 23:0 and 26:0, increased $(\mathrm{p}<0.04)$ over time during gestation. The percentage of palmitic acid (16:0) was not influenced ( $p>0.05)$ by diet and day, but there was a significant interaction $(p=0.02$, diet by day). On the other hand, 18:0 was decreased $(p=0.02)$ over time, regardless of diet; but the FMsupplemented diet did not make any greater reduction in 18:0 ( $\mathrm{p}=0.57$, diet by day). The percentage of total SFA was decreased $(p=0.001)$ over time but not influenced by the type of diet.

The effects of diet and day on Monounsaturated Fatty Acids (MUFA) are shown in Table 3 and 4. The FM-supplemented diet did not affect $(p>0.05)$ the average percentages of all MUFA (e.g., $9 c-16: 1$, $9 c-20: 1$, all trans isomers of $18: 1$ and all cis isomers of 18:1) compared to control except 11c-20:1. There was an increase $(\mathrm{p}<0.04)$ over time in $9 c-16: 1,11 t$ $18: 1,12 t-18: 1,13-14 t-18: 1,12 c-18: 1,13 c-18: 1$ and $16 t-18: 1$, regardless of diet. However, there was no significant interaction (diet by day) on these FA except 11t-18:1 $(\mathrm{p}=0.01)$. Type of diet and day did not change $(p>0.05)$ the average percentages of total trans-18:1 and total cis-18:1, although there was a significant interaction ( $\mathrm{p}=0.01$, diet by day) on total trans-18:1. There was no diet, day and/or diet by day interaction effect on oleic acid $(9 c-18: 1), 11 c-18: 1$ and 10t-18:1 and total MUFA.

The average percentage of linoleic acid (18:2n6) in ewe plasma of the FM-supplemented group was not changed $(\mathrm{p}=0.16)$ compared with the control group. However, this FA was increased $(\mathrm{p}=0.001)$ over time across both diets. The FM-supplementation did not change ( $p>0.05)$ the percentages of $20: 2 n 6$, 20:3n6, 20:4n6 and 22:5n6 in ewe plasma compared with control, but were affected $(\mathrm{p}<0.001)$ by day. The percentages of $\gamma$-linolenic acid (18:3n6), 22:2n6 and $22: 4 \mathrm{n} 6$ were not changed $(\mathrm{p}=0.08)$ by diet or day. The FM-supplementation did not change the content of total omega-6 PUFA compared to control, but the content was changed $(p=0.001)$ over time.

The percentages of $\alpha$-linolenic acid (18:3n 3$)$, $20: 3 n 3$ and $22: 5 n 3$ were changed $(p<0.05)$ over time, but were not altered by diet and diet by day interaction (Table 3). The FM-supplementation in the diet of ewes increased $(p<0.001)$ the percentages of eicosapentaenoic acid (20:5n3) and docosahexaenoic acid (22:6n3) compared to control. The contents of these FA were also increased $(\mathrm{p}<0.002)$ over time and were greater for FM-supplemented group ( $p<0.002$, diet by day interaction). Type of diet and day had significant influence $(p<0.03)$ on the percentages of total n3-PUFA and total VL_n3PUFA (20:5n3 plus 22:5n3 plus 22:6n3). There was also a significant interaction ( $\mathrm{p}<0.03$, diet by day) on total n3-PUFA and total VL_n3-PUFA.

The percentages of $9 c 11 t$-CLA, $9 t 11 c$-CLA, $10 t 12 c$-CLA, $9 t 11 t+10 t 12 t$-CLA and total CLA in plasma of ewes fed FM-supplemented diet were not changed compared to control Table 5. The contents of these CLA isomers were also not changed over time. On the other hand, the percentage of $11 t 13 t$ CLA was increased $(p=0.01)$ over time, regardless of diet; but there was no significant interaction ( $>>0.05$, diet by day).

Table 6 shows some selected FA in the plasma of lambs at birth from ewes fed either a control or FM-supplemented diet. The percentages of the main SFA, such as 16:0, 18:0, were not different ( $p>0.05)$ between the two treatments. Among MUFA (e.g., $10 t-18: 1, \quad 11 t-18: 1$ and $9 c-18: 1)$, there was an increase $(\mathrm{p}<0.05)$ in plasma $11 t-18: 1$ in lambs from the maternal FM-supplemented group compared with the control group. The percentages of PUFA, such as $18: 2 \mathrm{n} 6,18: 3 \mathrm{n} 3,20: 5 \mathrm{n} 3$ and $22: 6 \mathrm{n} 3$, were greater $(\mathrm{p}<0.05)$ in FM-supplemented group. The $9 c 11 t$-CLA isomer was greater $(\mathrm{p}<0.05)$ in lambs from the maternal FM-supplemented group compared with the control group, whereas no difference in $10 t 12 c$-CLA content between two treatment groups was observed. 
American J. Animal \& Vet. Sci., 7 (2): 67-74, 2012

Table 3: Least square means for fatty acids ( $\mathrm{g} / 100 \mathrm{~g}$ total FA) in the plasma of ewes fed a basal diet supplemented with either a control or fishmeal supplement during gestation ${ }^{1}$

\begin{tabular}{|c|c|c|c|c|c|c|c|c|c|c|c|c|c|c|}
\hline \multirow{2}{*}{$\begin{array}{l}\text { FA/day of } \\
\text { gestation }\end{array}$} & \multicolumn{4}{|c|}{ Control } & \multicolumn{4}{|c|}{ FM-supplemented } & \multirow{2}{*}{$\operatorname{SEM}(P$} & \multirow{2}{*}{$\begin{array}{c}\text { Diet } \\
\text {-value) }\end{array}$} & \multicolumn{2}{|c|}{$\begin{array}{l}\text { Day } \\
\text { (P- value) }\end{array}$} & \multicolumn{2}{|c|}{$\begin{array}{l}\text { Diet X Day } \\
(P \text {-value })\end{array}$} \\
\hline & $100 \mathrm{~d}$ & $114 \mathrm{~d}$ & $128 \mathrm{~d}$ & $142 \mathrm{~d}$ & $100 \mathrm{~d}$ & $114 \mathrm{~d}$ & $128 \mathrm{~d}$ & $142 \mathrm{~d}$ & & & ${ }^{2} \mathrm{~L}$ & ${ }^{3} \mathrm{Q}$ & ${ }^{2} \mathrm{~L}$ & ${ }^{3} \mathrm{Q}$ \\
\hline 12:0 & 0.09 & 0.09 & 0.11 & 0.12 & 0.09 & 0.10 & 0.10 & 0.10 & 0.01 & 0.770 & 0.040 & 0.880 & 0.360 & 0.820 \\
\hline iso-13:0 & 0.04 & 0.04 & 0.03 & 0.03 & 0.04 & 0.04 & 0.03 & 0.02 & 0.00 & 0.440 & 0.002 & 0.160 & 0.890 & 0.780 \\
\hline anteiso-13:0 & 0.08 & 0.09 & 0.10 & 0.06 & 0.09 & 0.10 & 0.08 & 0.04 & 0.01 & 0.760 & 0.001 & 0.001 & 0.070 & 0.740 \\
\hline 13:0 & 0.03 & 0.04 & 0.04 & 0.03 & 0.04 & 0.04 & 0.04 & 0.03 & 0.01 & 0.820 & 0.550 & 0.040 & 0.320 & 0.870 \\
\hline iso-14:0 & 0.16 & 0.17 & 0.20 & 0.18 & 0.16 & 0.17 & 0.20 & 0.15 & 0.03 & 0.850 & 0.630 & 0.090 & 0.640 & 0.550 \\
\hline 14:0 & 0.56 & 0.59 & 0.61 & 0.66 & 0.52 & 0.55 & 0.71 & 0.66 & 0.07 & 0.990 & 0.030 & 0.680 & 0.490 & 0.390 \\
\hline iso-15:0 & 0.42 & 0.44 & 0.36 & 0.32 & 0.39 & 0.41 & 0.35 & 0.30 & 0.06 & 0.760 & 0.010 & 0.030 & 0.760 & 0.890 \\
\hline anteiso-15:0 & 0.54 & 0.57 & 0.45 & 0.42 & 0.52 & 0.54 & & 0.41 & 0.08 & 0.910 & 0.010 & & 0.560 & 0.130 \\
\hline 15:0 & 1.00 & 1.05 & 0.98 & 0.84 & 0.94 & 0.99 & 1.14 & 0.82 & 0.14 & 0.970 & 0.140 & 0.001 & 0.510 & 0.190 \\
\hline iso-16:0 & 0.62 & 0.65 & 0.76 & 0.64 & 0.57 & 0.60 & 0.90 & 0.62 & 0.09 & 0.980 & 0.120 & 0.002 & 0.490 & 0.110 \\
\hline $16: 0$ & 17.40 & 16.90 & 15.60 & 15.60 & 15.70 & 16.10 & 16.40 & 16.20 & 0.52 & 0.650 & 0.160 & 0.870 & 0.020 & 0.240 \\
\hline iso-17:0 & 0.83 & 0.87 & 0.71 & 0.61 & 0.77 & 0.81 & 0.68 & 0.60 & 0.06 & 0.530 & 0.001 & 0.010 & 0.560 & 0.800 \\
\hline anteiso-17:0 & 0.89 & 0.93 & 0.95 & 0.84 & 0.89 & 0.93 & 1.25 & 0.71 & 0.11 & 0.650 & 0.600 & 0.010 & 0.870 & 0.140 \\
\hline $9 c-16: 1$ & 0.35 & 0.37 & 0.44 & 0.45 & 0.35 & 0.37 & 0.41 & 0.38 & 0.05 & 0.720 & 0.040 & 0.330 & 0.250 & 0.560 \\
\hline 17:0 & 1.40 & 1.48 & 1.42 & 1.25 & 1.35 & 1.42 & 1.53 & 1.26 & 0.08 & 0.990 & 0.160 & 0.001 & 0.450 & 0.510 \\
\hline iso-18:0 & 0.20 & 0.21 & 0.19 & 0.20 & 0.22 & 0.24 & 0.18 & 0.21 & 0.03 & 0.720 & 0.430 & 0.870 & 0.510 & 0.660 \\
\hline anteiso-18:0 & 0.35 & 0.37 & 0.44 & 0.40 & 0.37 & 0.38 & 0.29 & 0.27 & 0.04 & 0.290 & 0.470 & 0.100 & 0.010 & 0.750 \\
\hline 18:0 & 24.30 & 21.80 & 20.20 & 21.50 & 26.30 & 22.90 & 20.70 & 21.90 & 1.74 & 0.640 & 0.020 & 0.010 & 0.570 & 0.800 \\
\hline $9 t, 12 c-18: 2$ & 0.09 & 0.09 & 0.11 & 0.07 & 0.08 & 0.08 & 0.13 & 0.07 & 0.01 & 0.760 & 0.770 & 0.001 & 0.290 & 0.220 \\
\hline $11 t, 15 c-18: 2$ & 0.13 & 0.14 & 0.16 & 0.07 & 0.09 & 0.09 & 0.12 & 0.08 & 0.03 & 0.330 & 0.360 & 0.060 & 0.310 & 0.470 \\
\hline $18: 2 \mathrm{n} 6$ & 18.90 & 19.90 & 25.20 & 24.00 & 19.00 & 20.60 & 21.00 & 21.80 & 0.92 & 0.160 & 0.001 & 0.180 & 0.070 & 0.550 \\
\hline $20: 0$ & 0.23 & 0.24 & 0.20 & 0.17 & 0.23 & & 0.50 & 0.26 & 0.04 & 0.010 & 0.790 & 0.010 & 0.090 & 0.020 \\
\hline $18: 3 n$ & 0.18 & 0.18 & 0.24 & 0.20 & & & & & 0.03 & & 0.940 & & 0 & 0.590 \\
\hline $9 c-20$ & 0.07 & 0.0 & & & & & & & & & & & & 0.360 \\
\hline $11 c-20: 1$ & 0.05 & 0.05 & 0.05 & 0.02 & 0.05 & & & & 0.01 & & 0.840 & & 0.070 & 0.070 \\
\hline $18: 3 n 3$ & 5.09 & 5.35 & 4.91 & 6.78 & 4.32 & 4.55 & 4.1 & 5.7 & 0.42 & 0.120 & 0.002 & 0.001 & 0.760 & 0.760 \\
\hline 21:0 & 0.02 & 0.03 & 0.01 & 0.02 & 0.01 & 0.01 & 0.02 & 0.02 & 0.01 & 0.620 & 0.770 & 0.910 & 0.150 & 0.440 \\
\hline $20: 2 \mathrm{n} 6$ & 0.05 & 0.05 & 0.08 & 0.09 & 0.05 & 0.06 & 0.07 & 0.09 & 0.01 & 0.950 & 0.001 & 0.030 & 0.520 & 0.610 \\
\hline 22:0 & 0.15 & 0.16 & 0.11 & 0.12 & 0.12 & 0.16 & 0.24 & 0.16 & 0.03 & 0.270 & 0.880 & 0.190 & 0.120 & 0.220 \\
\hline $20: 3 \mathrm{n} 6$ & 0.22 & 0.23 & 0.38 & 0.39 & 0.22 & 0.23 & 0.25 & 0.22 & 0.03 & 0.080 & 0.001 & 0.310 & 0.001 & 0.520 \\
\hline $20: 3 \mathrm{n} 3$ & 0.05 & 0.06 & 0.08 & 0.07 & 0.05 & 0.05 & 0.10 & 0.10 & 0.02 & 0.520 & 0.010 & 0.790 & 0.280 & 0.910 \\
\hline $20: 4 n 6$ & 2.90 & 3.05 & 2.44 & 2.26 & 3.17 & 3.33 & 2.50 & 2.22 & 0.28 & 0.720 & 0.001 & 0.010 & 0.290 & 0.690 \\
\hline 23:0 & 0.12 & 0.12 & 0.09 & 0.02 & 0.08 & 0.09 & 0.11 & 0.02 & 0.02 & 0.460 & 0.001 & 0.002 & 0.230 & 0.730 \\
\hline $22: 2 \mathrm{n} 6$ & 0.03 & 0.03 & 0.02 & 0.03 & 0.02 & 0.02 & 0.03 & 0.03 & 0.01 & 0.770 & 0.370 & 0.170 & 0.220 & 0.330 \\
\hline $20: 5 \mathrm{n} 3$ & 1.03 & 1.09 & 0.93 & 1.08 & 1.07 & 1.79 & 2.07 & 1.83 & 0.12 & 0.001 & 0.002 & 0.002 & 0.002 & 0.001 \\
\hline 24:0 & 0.15 & 0.16 & 0.11 & 0.10 & 0.13 & 0.13 & 0.14 & 0.10 & 0.03 & 0.930 & 0.070 & 0.160 & 0.350 & 0.710 \\
\hline $22: 3 \mathrm{n} 3$ & 0.05 & 0.05 & 0.02 & 0.02 & 0.05 & 0.0 & 0.04 & 0.0 & 0.01 & 0.280 & 0.001 & 0.080 & 0.030 & 0.370 \\
\hline $22: 4 n 6$ & 0.10 & 0.11 & 0.16 & 0.1 & 0.11 & & 0. & 0. & 0.0 & 0.0 & 0.350 & 0.580 & 0.001 & 0.430 \\
\hline $22: 5 n 6$ & & 0.25 & & 0.0 & & & & & & & & & & 0.810 \\
\hline $22: 5 n 3$ & & 1.91 & & 2.2 & & & & & & & & & 20 & 0.240 \\
\hline $26: 0$ & 0.20 & 0.21 & 0.09 & 0.11 & 0.24 & 0.25 & 0.14 & 0.10 & 0.03 & 0.370 & & 0.360 & 0.540 & 0.2400 \\
\hline $22: 6 n 3$ & 1.51 & 1.58 & 1.32 & 1.34 & 1.62 & 2.93 & 3.41 & 3.44 & 0.21 & 0.001 & 0.001 & 0.010 & 0.001 & 0.010 \\
\hline${ }^{4}$ Total SFA & 49.70 & 47.10 & 43.80 & 44.20 & 49.60 & 47.20 & 46.30 & 44.90 & 1.48 & 0.590 & 0.001 & 0.250 & 0.640 & 0.540 \\
\hline${ }^{5}$ Total MUFA & 17.40 & 18.30 & 17.70 & 16.50 & 18.00 & 16.20 & 17.20 & 16.90 & 0.99 & 0.720 & 0.350 & 0.770 & 0.880 & 0.080 \\
\hline${ }^{6}$ Total n6 PUFA & 22.70 & 23.90 & 28.70 & 27.20 & 22.91 & 24.70 & 24.40 & 24.70 & 1.04 & 0.200 & 0.001 & 0.090 & 0.060 & 0.630 \\
\hline${ }^{7}$ Total n3 PuFA & 9.54 & 10.00 & 9.22 & 11.56 & 8.94 & 11.31 & 11.62 & 13.07 & 0.47 & 0.030 & 0.001 & 0.440 & 0.030 & 0.030 \\
\hline${ }^{8}$ Total CLA & 0.47 & 0.50 & 0.45 & 0.46 & 0.40 & 0.43 & 0.43 & 0.41 & 0.03 & 0.240 & 0.620 & 0.200 & 0.420 & 0.5700 \\
\hline Total trans-18:1 & 3.05 & 3.21 & 2.75 & 2.46 & 2.43 & 2.55 & 4.20 & 3.25 & 0.46 & 0.690 & 0.410 & 0.020 & 0.010 & 0.310 \\
\hline Total cis-18:1 & 13.90 & 14.60 & 14.40 & 13.48 & 15.09 & 13.14 & 12.26 & 13.11 & 1.08 & 0.600 & 0.190 & 0.530 & 0.380 & 0.030 \\
\hline $\begin{array}{l}{ }^{9} \text { Total VL_ } \\
\text { n3-PUFA }\end{array}$ & 4.35 & 4.58 & 4.22 & 4.69 & 4.52 & 6.65 & 7.32 & 7.17 & 0.40 & 0.010 & 0.001 & 0.010 & 0.001 & 0.001 \\
\hline
\end{tabular}

${ }^{\mathrm{T}}$ Average of 4 measurements; ${ }^{2} \mathrm{~L}$ : linear effect ; ${ }^{3} \mathrm{Q}$ : quadratic effect; ${ }^{4}$ Total SFA: all saturated fatty acids (without any double bond, 12:0 to 26:0).

${ }^{5}$ Total MUFA: all monounsaturated fatty acids with a single double bond (16:1 to 20:1). ${ }^{6}$ Total $\mathrm{n}-6$ polyunsaturated fatty acids (PUFA): 18:2n6; 18:3n6; 20:2n6; 20:3n6; 20:4n6; 22:2n6; 22:4n6; and 22:5n6. ${ }^{7}$ Total n-3 polyunsaturated fatty acids (PUFA): 18:3n3; 20:3n3; 20:5n3; 22:3n3; 22:5n3; and 22:6n3. ${ }^{8}$ Total conjugated linoleic acid (CLA): $9 c, 11 t 18: 2 ; 9 t, 11 c 18: 2 ; 10 t, 12 c 18: 2 ; 11 t 13 t 18: 2 ;$ and $9 \mathrm{t}, 11 t$ plus $10 t 12 t 18: 2 .{ }^{9}$ Total very long chain n-3 PUFA (VL_n3-PUFA, >18C): 20:5n3; 22:5n3; and 22:6n3 
American J. Animal \& Vet. Sci., 7 (2): 67-74, 2012

Table 4: Positional isomers of 18:1 monoenes ( $\mathrm{g} / 100 \mathrm{~g}$ total FA) in the plasma of ewes fed a basal diet supplemented with either a control or fishmeal supplement during gestation

\begin{tabular}{|c|c|c|c|c|c|c|c|c|c|c|c|c|c|c|}
\hline \multirow[b]{2}{*}{$\begin{array}{l}\text { FA / day } \\
\text { of gestation }\end{array}$} & \multicolumn{4}{|c|}{ Control } & \multicolumn{4}{|c|}{ FM-supplemented } & \multirow[b]{2}{*}{ SEM } & \multirow[b]{2}{*}{$\begin{array}{l}\text { Diet } \\
(P \text {-value })\end{array}$} & \multicolumn{2}{|c|}{$\begin{array}{l}\text { Day Diet } \\
(P \text {-value })\end{array}$} & \multicolumn{2}{|c|}{$\begin{array}{l}\text { X Day } \\
(P \text {-value })\end{array}$} \\
\hline & $100 \mathrm{~d}$ & $114 \mathrm{~d}$ & $128 \mathrm{~d}$ & $142 \mathrm{~d}$ & $100 \mathrm{~d}$ & $114 \mathrm{~d}$ & $128 \mathrm{~d}$ & $142 \mathrm{~d}$ & & & ${ }^{2} \mathrm{~L}$ & ${ }^{3} \mathrm{Q}$ & ${ }^{2} \mathrm{~L}$ & ${ }^{3} \mathrm{Q}$ \\
\hline $4 t-18: 1$ & 0.01 & 0.02 & 0.06 & 0.05 & 0.03 & 0.03 & 0.02 & 0.04 & 0.02 & 0.93 & 0.120 & 0.820 & 0.26 & 0.62 \\
\hline $5 t-18: 1$ & 0.05 & 0.05 & 0.07 & 0.03 & 0.05 & 0.06 & 0.10 & 0.03 & 0.02 & 0.51 & 0.560 & 0.030 & 0.85 & 0.52 \\
\hline $6-8 t-18: 1$ & 0.18 & 0.19 & 0.18 & 0.21 & 0.18 & 0.19 & 0.23 & 0.22 & 0.03 & 0.70 & 0.150 & 0.760 & 0.71 & 0.36 \\
\hline $9 t-18: 1$ & 0.26 & 0.28 & 0.24 & 0.21 & 0.24 & 0.25 & 0.30 & 0.24 & 0.02 & 0.74 & 0.230 & 0.010 & 0.10 & 0.41 \\
\hline $10 t-18: 1$ & 0.18 & 0.19 & 0.18 & 0.18 & 0.19 & 0.20 & 0.27 & 0.23 & 0.03 & 0.36 & 0.290 & 0.190 & 0.31 & 0.31 \\
\hline $11 t-18: 1$ & 1.78 & 1.87 & 1.10 & 0.93 & 1.19 & 1.26 & 1.67 & 1.22 & 0.25 & 0.79 & 0.040 & 0.050 & 0.01 & 0.49 \\
\hline $12 t-18: 1$ & 0.25 & 0.26 & 0.32 & 0.27 & 0.22 & 0.23 & 0.58 & 0.48 & 0.06 & 0.20 & 0.002 & 0.110 & 0.01 & 0.68 \\
\hline $13-14 t-18: 1$ & 0.23 & 0.24 & 0.39 & 0.38 & 0.20 & 0.21 & 0.74 & 0.53 & 0.10 & 0.32 & 0.001 & 0.220 & 0.13 & 0.32 \\
\hline $9 c-18: 1$ & 12.92 & 13.59 & 12.89 & 12.16 & 14.08 & 12.07 & 10.62 & 11.83 & 1.09 & 0.58 & 0.080 & 0.320 & 0.39 & 0.02 \\
\hline $11 c-18: 1$ & 0.70 & 0.74 & 0.80 & 0.84 & 0.74 & 0.78 & 0.90 & 0.79 & 0.06 & 0.66 & 0.070 & 0.260 & 0.56 & 0.22 \\
\hline $12 c-18: 1$ & 0.22 & 0.23 & 0.67 & 0.43 & 0.24 & 0.25 & 0.68 & 0.42 & 0.05 & 0.91 & 0.001 & 0.001 & 0.75 & 0.78 \\
\hline $13 c-18: 1$ & 0.04 & 0.04 & 0.04 & 0.04 & 0.04 & 0.04 & 0.06 & 0.08 & 0.01 & 0.16 & 0.001 & 0.150 & 0.00 & 0.31 \\
\hline $16 t-18: 1$ & 0.12 & 0.12 & 0.21 & 0.20 & 0.10 & 0.11 & 0.30 & 0.27 & 0.04 & 0.48 & 0.001 & 0.440 & 0.09 & 0.77 \\
\hline
\end{tabular}

${ }^{1}$ Average of 4 measurements; ${ }^{2} \mathrm{~L}$ : linear effect; ${ }^{3} \mathrm{Q}$ : quadratic effect

Table 5: Positional isomers of conjugated linoleic acid (g/100 g total FA) in the plasma of ewes fed a basal diet supplemented with either a control or fishmeal supplement during gestation ${ }^{1}$

\begin{tabular}{|c|c|c|c|c|c|c|c|c|c|c|c|c|c|c|}
\hline \multirow[b]{2}{*}{ FA / day of gestation } & \multicolumn{4}{|c|}{ Control } & \multicolumn{4}{|c|}{ FM-supplemented } & \multirow[b]{2}{*}{ SEM } & \multirow{2}{*}{$\begin{array}{l}\text { Diet } \\
\text { ( } P \text {-value })\end{array}$} & \multicolumn{2}{|c|}{$\begin{array}{l}\text { Day } \\
(P \text {-value })\end{array}$} & \multicolumn{2}{|c|}{$\begin{array}{l}\text { Diet X Day } \\
\text { ( } P \text {-value })\end{array}$} \\
\hline & $100 \mathrm{~d}$ & $114 \mathrm{~d}$ & $128 \mathrm{~d}$ & $142 \mathrm{~d}$ & $100 \mathrm{~d}$ & $114 \mathrm{~d}$ & $128 \mathrm{~d}$ & $142 \mathrm{~d}$ & & & ${ }^{2} \mathrm{~L}$ & ${ }^{3} \mathrm{Q}$ & ${ }^{2} \mathrm{~L}$ & ${ }^{3} \mathrm{Q}$ \\
\hline $9 c, 11 t$-CLA & 0.27 & 0.28 & 0.26 & 0.26 & 0.25 & 0.26 & 0.24 & 0.26 & 0.02 & 0.60 & 0.78 & 0.80 & 0.56 & 0.63 \\
\hline $9 t, 11 c-$ CLA & 0.02 & 0.02 & 0.01 & 0.03 & 0.01 & 0.01 & 0.01 & 0.02 & 0.00 & 0.29 & 0.09 & 0.07 & 0.96 & 0.22 \\
\hline $10 t, 12 c$-CLA & 0.02 & 0.03 & 0.02 & 0.02 & 0.02 & 0.02 & 0.04 & 0.01 & 0.01 & 0.97 & 0.52 & 0.16 & 0.71 & 0.16 \\
\hline $11 t, 13 t-$ CLA & 0.05 & 0.06 & 0.05 & 0.04 & 0.05 & 0.05 & 0.04 & 0.04 & 0.01 & 0.31 & 0.01 & 0.06 & 0.68 & 0.41 \\
\hline $9 t, 11 t+10 t, 12 t-$ CLA & 0.11 & 0.12 & 0.11 & 0.12 & 0.08 & 0.09 & 0.10 & 0.09 & 0.01 & 0.17 & 0.58 & 0.30 & 0.75 & 0.47 \\
\hline
\end{tabular}

${ }^{1}$ Average of 4 measurements; ${ }^{2} \mathrm{~L}$ : linear effect; ${ }^{3} \mathrm{Q}$ : quadratic effect

Table 6: Some selected fatty acids ( $\mathrm{g} / 100 \mathrm{~g} \mathrm{FA}$ ) in the plasma of lambs at birth from ewes fed a basal diet supplemented with either a control or fishmeal supplement ${ }^{1}$

\begin{tabular}{lccc}
\hline Fatty acid & \multicolumn{2}{c}{ Control FM-supplemented } & SEM \\
\hline $16: 0$ & 26.40 & 24.40 & 1.020 \\
$9 c-16: 1$ & 3.08 & 2.25 & 0.140 \\
$18: 0$ & 11.80 & 14.00 & 0.850 \\
$10 t-18: 1$ & 0.13 & 0.14 & 0.080 \\
$11 t-18: 1$ & $0.39^{\mathrm{a}}$ & $0.49^{\mathrm{b}}$ & 0.100 \\
$9 c-18: 1$ & $34.30^{\mathrm{a}}$ & 32.60 & 1.480 \\
$18: 2 \mathrm{n} 6$ & $2.86^{\mathrm{a}}$ & $3.93^{\mathrm{b}}$ & 1.080 \\
$18: 3 \mathrm{n} 3$ & $0.99^{\mathrm{a}}$ & $1.30^{\mathrm{b}}$ & 0.260 \\
$9 c, 11 t$-CLA & $0.25^{\mathrm{a}}$ & $0.36^{\mathrm{b}}$ & 0.050 \\
$10 t, 12 c-$-CLA & $0.01^{\mathrm{a}}$ & $0.01^{\mathrm{b}}$ & 0.003 \\
$20: 5 \mathrm{n} 3$ & $0.39^{\mathrm{a}}$ & $0.70^{\mathrm{b}}$ & 0.110 \\
$22: 6 \mathrm{n} 3$ & $0.93^{\mathrm{a}}$ & $1.59^{\mathrm{b}}$ & 0.150 \\
Total VL_n3-PUFA & $2.05^{\mathrm{a}}$ & $3.27^{\mathrm{b}}$ & 0.270 \\
\hline
\end{tabular}

${ }^{1}$ Average of 6 measurements; ${ }^{2}$ Total very long chain $\mathrm{n}-3$ PUFA (VL_n3-PUFA, >18C): 20:5n3; 22:5n3; and 22:6n3; ${ }^{\text {,b }}$ Means within a row with different superscripts differ $(\mathrm{p}<0.05)$

\section{DISCUSSION}

The aim of the current study was to establish whether feeding a diet supplemented with FM to ewes during gestation resulted in changes in the FA composition of their own plasma and subsequently in their lamb's plasma at birth. The major SFA in ewe plasma collected from both groups, were 16:0, 18:0,
$9 c-18: 1,18: 2 \mathrm{n} 6$ and 18:3n3, which was consistent with the results of Leat (1966). In the present study, the isomers of trans-18:1 FA and 9c11t-CLA in eweplasma were not increased with the supply of the FMsupplemented diet. It was reported that the proportion of plasma 18:3n 3 was higher in sheep supplemented with fish oil compared to control (Sinclair et al., 2005; Capper et al., 2006). According to them, tissue elongation and desaturation of $18: 3 \mathrm{n} 3$ to $20: 5 \mathrm{n} 3$ and 22:6n3 in sheep was inhibited by the VL_n3-PUFA, such as DHA and EPA, in fish oil. However, we did not find any significant difference in ewe plasma 18:3n3, when sheep were fed a FM-supplemented diet compared to control.

The FM-supplementation to ewes, in the present research, increased plasma $22: 6 \mathrm{n} 3$ by 3.3 times and $20: 5 \mathrm{n} 3$ by 1.8 times compared to control. The significant increase in plasma 20:5n 3 and 22:6n 3 in ewes offered diets containing fishmeal were a direct result of the increased dietary supply of preformed VL_n3-PUFA and in agreement with Capper et al. (2007). However, our study was carried out with a limited number of experimental units. The plasma of the control group also contained 20:5n 3 and 22:6n 3 , but in much lower percentages compared to FMsupplemented group. Endogenous synthesis of 20:5n3 
and $22: 6 n 3$ from $18: 3 n 3$ likely occurs to a limited extent (Wachira et al., 2002; Chikunya et al., 2004). The present study also revealed that the proportions of total VL_n3-PUFA in ewe plasma were increased and the proportions of total n6-PUFA were decreased by feeding the FM-supplemented diet. In an experiment conducted by Rooke et al. (1998), feeding tuna oil to sows increased the VL_n3-PUFA, especially 20:5n3 and 22:6n3, primarily at the expense of 18:2n6.

Although maternal ewe-plasma contained large amounts of 18:2n6 and a considerable amount of $18: 3 \mathrm{n} 3$, very small amounts of these FA (15.8\% of $18: 2 \mathrm{n} 6$ and $19.2 \%$ of $18: 3 \mathrm{n} 3$ ) were present in the plasma of newborn lambs. The same phenomenon was also observed by Leat (1966) in calves and lambs. Leat (1966) had also shown that newborn piglets contained much more 18:2n6 in their plasma than did the newborn ruminant. It appeared that, in contrast with the nonruminant, the ruminant placenta was relatively impermeable to $18: 2 \mathrm{n} 6$ and 18:3n3 (Hansen et al., 1964; Leat, 1966). In addition, it has been shown that the placentas of rats, rabbits and sheep are relatively impermeable to plasma cholesterol and phospholipids, whereas plasma non-esterified FA can pass the placental barrier into the fetus (Duyne et al., 1960; Popjak, 1954; McBride and Korn, 1964). Leat (1966) reported that sow-plasma non-esterified FA contained 4.8-10:0 times more 18:2n6 than ruminants and suggested that less $18: 2 \mathrm{n} 6$ acid would pass into the ruminant fetus than into the non-ruminant fetus in this way. It was also observed that supplementing diets of ruminants with protected PUFA during late gestation would improve the essential FA status of newborn ruminants (Soares, 1986). In the diet of ruminants, protected lipids containing PUFA can by-pass the rumen without biohydrogenation and concomitantly increase the concentration in maternal plasma as well as the fetus (Scott et al., 1971; Soares, 1986; Hobson and Stewart, 1997). In an experiment, Capper et al. (2006) found that lambs with increased plasma status of 20:5n3 and 22:6n3 had reduced latency of standing and suckling, which may have beneficial effects on lamb survival rate. According to O'Connor and Lawrence (1992) and Capper et al. (2006), the reduced latency of suckling might be due in part to increased visual acuity, which improved the ability of the lambs to successfully locate the udder. Our study confirmed that the plasma of lambs born to ewes fed the FM-supplemented diets during late gestation was characterized by high content of 20:5n3 and 22:6n3 compared with plasma obtained from the lambs born to the ewes that received control diet. However, further work is required to determine the survival rate of lambs born to ewes supplemented with FM. On the other hand, the present study revealed that the contents of $20: 5 n 3$ and $22: 6 n 3$ were enhanced in lambs born to ewes fed a FM-supplemented diet, suggesting specific FA can preferentially cross the placenta in late pregnancy.

\section{CONCLUSION}

The present study has clearly shown that when ewes are fed a FM-supplemented diet during late gestation, the percentages of EPA (20:5n3) and DHA (20:6n3) are significantly increased compared to control. Lambs born to FM-supplemented ewes had greater plasma concentrations of EPA, DHA and VL_n3-PUFA than lambs born to control ewes.

\section{ACKNOWLEDGEMENT}

The researcher would like to thank the staff at the Ponsonby Sheep Research Station, University of Guelph, for their technical assistance. Financial support from the Ontario Ministry of Agriculture, Food and Rural Affairs (OMAFRA); the Ontario Sheep Marketing Agency (OSMA); and the Natural Sciences and Engineering Research Council of Canada (BWM), are gratefully acknowledged.

\section{REFERENCES}

AOAC, 1996. Official Methods of Analysis. 16th Edn., Association of Official Analytical Chemists, Arlington, VA.

Bligh, E.G. and W.J. Dyer, 1959. A rapid method of total lipid extraction and purification. Can. J. Biochem. Physiol., 37: 911-917. PMID: 13671378

Brenna, J.T. and A. Lapillonne, 2009. Background paper on fat and fatty acid requirements during pregnancy and lactation. Ann. Nutr. Metab., 55: 97-122. PMID: 19752538

Capper, J.L., R.G. Wilkinson, A.M. Mackenzie and L.A. Sinclair, 2006. Polyunsaturated fatty acid supplementation during pregnancy alters neonatal behavior in sheep. J. Nutr., 136: 397-403. http://jn.nutrition.org/content/136/2/397.full

Capper, J.L., R.G. Wilkinson, A.M. Mackenzie and L.A. Sinclair, 2007. The effect of fish oil supplementation of pregnant and lactating ewes on milk production and lamb performance. Animal, 1: 889-898. DOI: $10.1017 /$ S1751731107000067

Cattaneo, D., V. Dell'Orto, G. Varisco, A. Agazzi and G. Savoini, 2006. Enrichment in n-3 fatty acids of goat's colostrum milk by maternal fish oil supplementation. Small Ruminant Res., 64: 22-29. DOI: 10.1016/j.smallrumres.2005.03.013

CCAC, 1993. Guide to the Care and Use of Experimental Animals. 2nd Edn., Canadian Council on Animal Care, ISBN-10: 0919087183 pp: 212. 
Chikunya, S., G. Demirel, M. Enser, J.D. Wood and R.G. Wilkinson et al., 2004. Biohydrogenation of dietary n-3 PUFA and stability of ingested vitamin $E$ in the rumen and their effects on microbial activity in sheep. Br. J. Nutr., 91: 539-550. PMID: 15035681

Cruz-Hernandez, C., Z. Deng, J. Zhou, A. R. Hill and M.P. Yurawecz et al., 2004. Methods for analysis of conjugated linoleic acids and trans-18:1 isomers in dairy fats by using a combination of gas chromatography, silver-ion thin-layer chromatography/gas chromatography and silverion liquid chromatography. J. AOAC Internat., 87: 545-562. PMID: 15164853

Duyne, C.M.V., H. R. Parker, R.J. Havel and L.W. Holm, 1960. Free fatty acid metabolism in fetal and newborn sheep. Am. J. Physiol., 199: 987-990.

Hansen, A.E., H.F. Wiese, D.J. Adam, A.N. Boelsche and M.E. Haggard et al., 1964. Influence of diet on blood serum lipids in pregnant women and newborn infants. Am. J. Clin. Nutr., 15: 11-19. PMID: 14192741

Hobson, P.N. and C.S. Stewart, 1997. The Rumen Microbial Ecosystem. 2nd Edn., Blackie Academic and Professional Press, London, pp: 719. ISBN-10: 0751403660

Hsieh, A.T. and J.T. Brenna, 2009. Dietary docosahexaenoic acid but not arachidonic acid influences central nervous system fatty acid status in baboon neonates. Prostaglandins Leukot. Essent. Fatty Acids, 81: 105-110. PMID: 19524425

Kramer, J. K., C. B. Blackadar and J. Zhou. 2002. Evaluation of two GC columns (60-m SUPELCOWAX 10 and 100-m CP Sil 88) for analysis of milk fat with emphasis on CLA, 18:1, $18: 2$ and 18:3 isomers and short- and long-chain FA. Lipids 37:823-835.

Leat, W.M.F., 1966. Fatty acid composition of the plasma lipids of newborn and maternal ruminants. Biochem. J., 98: 598-603. PMID: 5949570

Littell, R.C., G.A. Milliken, W.W. Stroup and R.D. Wolfinger, 1996. SAS system for mixed models. 1st Edn., SAS Institute Inc., Carym, NC, ISBN-10: $1555447791, \mathrm{pp}: 656$.

McBride, O.W. and E.D. Korn, 1964. Uptake of free fatty acids and chylomicron glycerides by guinea pig mammary gland in pregnancy and lactation. J. Lipid Res., 5: 453-458. PMID: 5896034

Muller, A., R. Ringseis, K. Düsterloh, S. Gahler and K. Eder et al., 2005. Detection of conjugated dienoic fatty acids in human vascular smooth muscle cells treated with conjugated linoleic acid. Biochim. Biophys. Acta, 1737: 145-151. PMID: 16275160
Noble, R.C., J.H. Shand, J.T. Drummond and J.H. Moore, 1978. "Protected" polyunsaturated fatty acid in the diet of the ewe and the essential fatty acid status of the neonatal lamb. J. Nutr., 108: 1868-76. PMID: 712431

O'Connor, C.E. and A.B. Lawrence, 1992. Relationship between lamb vigour and ewe behaviour at parturition. Anim. Prod., 54: 361-366. .DOI: 10.1017/S000335610002081X

Odongo, N.E., M.M. Or-Rashid, E. Kebreab, J. France and B.W. McBride, 2007. Effect of supplementing myristic acid in dairy cow rations on ruminal methanogenesis and fatty acid profile in milk. J. Dairy Sci., 90: 1851-1858. PMID: 17369226

Popjak, G., 1954. The origin of fetal lipids. Cold Spring Harb. Symp. Quant. Biol., 19: 200-208. PMID: 13291204

Rooke, J.A., I.M. Bland and S.A. Edward, 1998. Effect of feeding tuna oil or soyabean oil as supplements to sows in late pregnancy on piglet tissue composition and viability. Br. J. Nutr., 80: 273-80. PMID: 9875067

Saldanha, L.G., N.S Jr. and J.T. Brenna, 2009. Prostaglandins Leukot. Essent. Fatty Acids, 81: 233-236.

Scott, T.W., L.J. Cook and S.C. Mills, 1971. Protection of dietary polyunsaturated fatty acids against microbial hydrogenation in ruminants. J. Am. Oil Chem. Soc., 48: 358-364. DOI: 10.1007/BF02890762

Sinclair, L.A., S.L. Cooper, S. Chikunya, R.G. Wilkinson and K.G. Hallett et al., 2005. Biohydrogenation of $\mathrm{n}-3$ polyunsaturated fatty acids in the rumen and their effects on microbial metabolism and plasma fatty acid concentrations in sheep. Anim. Sci., 81: 239-48. DOI: 10.1079/ASC50040239

Singer, D., 1998. Thermometry calorimetry in the recent advances in monitoring research. Thermochimica Acta., 309: 39-47. DOI: 10.1016/S0040-6031(97)00427-9

Soares, M.C., 1986. Effect of dietary protected lipids on the essential fatty acid status of the newborn kid. J. Nutr., 116: 1473-1479. PMID: 3761003

Wachira, A.M., L.A. Sinclair, R.G. Wilkinson, M. Enser and J.D. Wood et al., 2002. Effects of dietary fat source and breed on the carcass composition, n-3 polyunsaturated fatty acid and conjugated linoleic acid content of sheep meat and adipose tissu. Br. J. Nutr., 88: 697-709. PMID: 12493092

Williams, C.M., 2000. Dietary fatty acids and human health. Ann. Zootech., 49: 165-180. DOI: 10.1051/animres:2000116 